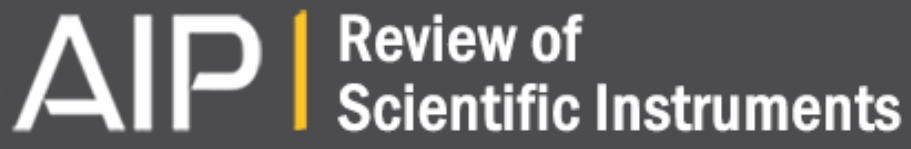

High field electron paramagnetic resonance spectroscopy under ultrahigh vacuum conditions-A multipurpose machine to study paramagnetic species on well defined single crystal surfaces

J. Rocker, D. Cornu, E. Kieseritzky, A. Seiler, O. Bondarchuk, W. Hänsel-Ziegler, T. Risse, and H.-J. Freund

Citation: Review of Scientific Instruments 85, 083903 (2014); doi: 10.1063/1.4893729

View online: http://dx.doi.org/10.1063/1.4893729

View Table of Contents: http://scitation.aip.org/content/aip/journal/rsi/85/8?ver=pdfcov

Published by the AIP Publishing

\section{Articles you may be interested in}

A quasioptical transient electron spin resonance spectrometer operating at 120 and $240 \mathrm{GHz}$

Rev. Sci. Instrum. 76, 074101 (2005); 10.1063/1.1942533

Axially uniform resonant cavity modes for potential use in electron paramagnetic resonance spectroscopy Rev. Sci. Instrum. 72, 4188 (2001); 10.1063/1.1405796

A "shunt" Fabry-Perot resonator for high-frequency electron spin resonance utilizing a variable coupling scheme Rev. Sci. Instrum. 69, 3022 (1998); 10.1063/1.1149050

System for electron paramagnetic resonance measurements at high pressure and low temperature Rev. Sci. Instrum. 68, 3877 (1997); 10.1063/1.1148041

Aqueous sample holders for high-frequency electron spin resonance

Rev. Sci. Instrum. 68, 2838 (1997); 10.1063/1.1148205

NEW

Model PS-100

Tabletop Cryogenic

Probe Station

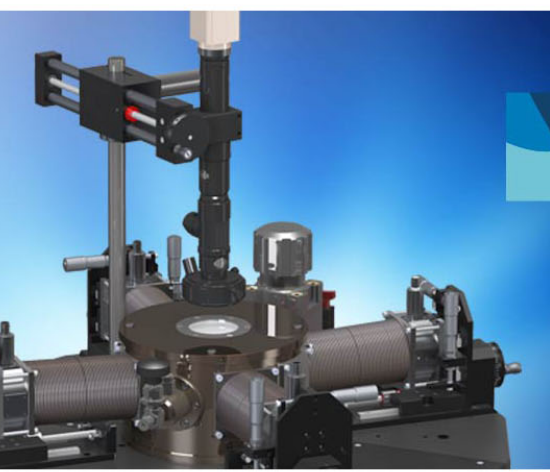

Lake Shore CRYOTRONICS

An affordable solution for a wide range of research 


\title{
High field electron paramagnetic resonance spectroscopy under ultrahigh vacuum conditions-A multipurpose machine to study paramagnetic species on well defined single crystal surfaces
}

\author{
J. Rocker, ${ }^{1}$ D. Cornu, ${ }^{1}$ E. Kieseritzky, ${ }^{1}$ A. Seiler, ${ }^{1,2}$ O. Bondarchuk, ${ }^{1,3}$ W. Hänsel-Ziegler, ${ }^{1}$ \\ T. Risse, ${ }^{1,4, a)}$ and H.-J. Freund ${ }^{1}$ \\ ${ }^{1}$ Fritz-Haber-Institut der MPG, Faradayweg 4-6, 14195 Berlin, Germany \\ ${ }^{2}$ Laboratorium für Applikationen der Synchrotronstrahlung, KIT Campus Süd, Kaiserstr. 12, \\ 76131 Karlsruhe, Germany \\ ${ }^{3}$ CIC energiGUNE, Parque Tecnologico, C/Albert Einstein 48, CP 01510 Minano (Alava), Spain \\ ${ }^{4}$ Institut für Chemie und Biochemie, Freie Universität Berlin, Takustr. 3, 14195 Berlin, Germany
}

(Received 10 June 2014; accepted 11 August 2014; published online 26 August 2014)

\begin{abstract}
A new ultrahigh vacuum (UHV) electron paramagnetic resonance (EPR) spectrometer operating at $94 \mathrm{GHz}$ to investigate paramagnetic centers on single crystal surfaces is described. It is particularly designed to study paramagnetic centers on well-defined model catalysts using epitaxial thin oxide films grown on metal single crystals. The EPR setup is based on a commercial Bruker E600 spectrometer, which is adapted to ultrahigh vacuum conditions using a home made Fabry Perot resonator. The key idea of the resonator is to use the planar metal single crystal required to grow the single crystalline oxide films as one of the mirrors of the resonator. EPR spectroscopy is solely sensitive to paramagnetic species, which are typically minority species in such a system. Hence, additional experimental characterization tools are required to allow for a comprehensive investigation of the surface. The apparatus includes a preparation chamber hosting equipment, which is required to prepare supported model catalysts. In addition, surface characterization tools such as low energy electron diffraction (LEED)/Auger spectroscopy, temperature programmed desorption (TPD), and infrared reflection absorption spectroscopy (IRAS) are available to characterize the surfaces. A second chamber used to perform EPR spectroscopy at $94 \mathrm{GHz}$ has a room temperature scanning tunneling microscope attached to it, which allows for real space structural characterization. The heart of the UHV adaptation of the EPR experiment is the sealing of the Fabry-Perot resonator against atmosphere. To this end it is possible to use a thin sapphire window glued to the backside of the coupling orifice of the Fabry Perot resonator. With the help of a variety of stabilization measures reducing vibrations as well as thermal drift it is possible to accumulate data for a time span, which is for low temperature measurements only limited by the amount of liquid helium. Test measurements show that the system can detect paramagnetic species with a density of approximately $5 \times 10^{11} \mathrm{spins} / \mathrm{cm}^{2}$, which is comparable to the limit obtained for the presently available UHV-EPR spectrometer operating at $10 \mathrm{GHz}$ (X-band). Investigation of electron trapped centers in $\mathrm{MgO}(001)$ films shows that the increased resolution offered by the experiments at W-band allows to identify new paramagnetic species, that cannot be differentiated with the currently available methodology. (C) 2014 AIP Publishing LLC. [http://dx.doi.org/10.1063/1.4893729]
\end{abstract}

\section{INTRODUCTION}

Motivated by the broad area of application including sensor technology, optoelectronics, or catalysis, the interest in a detailed description of microscopic processes on metal oxide surfaces has increased continuously in the past. In the field of heterogeneous catalysis metal oxides are either used as support for an active species - oftentimes a metal - or they serve as catalysts themselves. An understanding of the catalyst activity requires insight into the correlation between the catalytic properties and geometric and electronic structure at the atomic level. In case of complex powdered catalysts this ambitious goal is beyond today's experimental capabilities.

a) Author to whom correspondence should be addressed. Electronic mail: risse@chemie.fu-berlin.de
Thus, the quest for solutions to this problem is still actively pursued in various directions. The development of model catalysts, which go beyond the investigation of metal single crystal surfaces recognized by the 2007 Nobel-Price in chemistry to Gerhard Ertl, ${ }^{1}$ plays an important role in this respect as the metal nanoparticles respond to the environment, for example, the support, which is in turn coupled to their reactivity. Hence, controlling the environment and understanding the different effects at an atomistic level is an important goal. Model catalysts based on thin, single crystalline oxide films grown on metal single crystal surfaces were shown to be a valuable approach, because they allow to grasp considerable part of the complexity of real catalysts while being suitable to characterization using the methodology of modern surface science. ${ }^{2-4}$

The development of suitable model systems is an important ingredient to advance our understanding of such sys- 
tem. However, the availability of appropriate experimental techniques to address the various questions at hand is at least of equal importance. In fact, major advancements of our understanding are associated with the development of experimental techniques. Scanning tunneling microscopy is perhaps one of the best examples where a single technique has advanced our perception of surfaces tremendously. ${ }^{5}$ In comparison to metals, oxides have considerably different electronic properties, which also require different methodologies to probe them. Defects and in particular point defects may serve as an instructive example. The latter are thought to play an important role for the properties of oxide surfaces, however, an atomistic characterization is still challenging. This is the typical scenario where method development sets in. With respect to point defects in oxides electron paramagnetic resonance (EPR) spectroscopy was used for decades to characterize these species in the bulk, due to the paramagnetic nature of some of these sites (e.g., Ref. 6). For powdered material also surface species have been studied to quite some extent and combination with theory proved to be very valuable in assigning the experimental results to specific species. ${ }^{7-10}$ For such systems it is almost impossible to investigate surface centers in the absence of interacting molecules. This might only be achieved if the powder is created inside an ultrahigh vacuum (UHV) environment. ${ }^{11}$ The application of EPR spectroscopy in UHV and its combination to single crystal surfaces is more demanding, but was shown possible using X-Band $(9.5 \mathrm{GHz})$ spectroscopy. ${ }^{12-14}$ These experiments allow the characterization of paramagnetic surface species in terms of geometric as well as electronic environment. ${ }^{15-17}$ While cw-EPR spectroscopy at X-band is well established for the investigation of single crystal surfaces the spectral resolution is sometimes insufficient to discriminate between different sites. In case the spectral resolution is limited by g-anisotropy of the species, spectral resolution can be enhanced by increasing the operation frequency of the spectrometer along the lines pursued in NMR spectroscopy. In practice, the extension to higher frequencies proved useful not only in terms of spectral resolution but also in terms of sensitivity. ${ }^{18-20}$ Since the first high field EPR spectrometer installation in $1983^{21}$ the use of frequencies $\geq 94 \mathrm{GHz}$ has steadily increased and the first commercial W-band spectrometer $(94 \mathrm{GHz})$ in 1996 made this frequency regime accessible for a larger scientific group. ${ }^{22}$ Within this paper we want to describe the successful adaptation of such a commercially available W-Band (94 GHz) EPR spectrometer (E600, Bruker) to a multipurpose surface science apparatus. The increase of the operating frequency and thus the spectral resolution by an order of magnitude as compared to the existing UHV compatible X-band spectrometers has severe implications for the experimental implementation. A simple adaptation of the X-band design based on a monomodal resonator operating at ambient pressure and a quartz insert connected via a glass to metal seal to the UHV apparatus to the dimensions of W-band radiation would lead to sample sizes of a few hundred micrometers. Apart from tremendous technical problems in handling samples of that size under UHV conditions, such samples would be incompatible with most standard surface science tools. However, EPR spectroscopy is not an inherently surface sensitive method and is limited to the investigation of paramagnetic species, which are typically minority species on the surface. Therefore, a proper characterization of the surface by other methods is mandatory. The key idea to circumvent these complications is to use a multimodal Fabry-Perot type of resonator, which has been successfully developed and used for W-Band EPR spectroscopy in various laboratories (e.g., Refs. 23-25). The planar metal single crystal used as substrate for the growth of the single crystalline oxide film will serve as one mirror of the Fabry Perot resonator. The second curved mirror will be used to connect to the microwave bridge. ${ }^{26}$ While the principle idea is straightforward the details of the technical realization requires some attention and will be discussed in detail below.

The paper is structured as follows: Sec. II describes the experimental setup focusing on the design of the chambers and different techniques implemented to allow for a comprehensive characterization of the surfaces to be investigated. The details of the implementation of the EPR spectrometer in particular the details of Fabry-Perot resonator to measure W-band EPR spectroscopy under UHV conditions will be discussed in Sec III. Section IV will present initial experiments on thin $\mathrm{MgO}(001)$ films and discuss the results in light of the experiments obtained at X-band on similar systems.

\section{SYSTEM DESIGN}

The investigation of well-defined model catalysts requires an in situ preparation of the sample. As EPR spectroscopy is solely sensitive to paramagnetic species additional techniques to characterize the properties of the sample are needed to ensure well-defined surface properties, which are a prerequisite to interpret the EPR data appropriately. Geometric structure is of particular importance, however, other information such as the properties of adsorbed gases play an important role, too. The latter information is not only interesting to investigate the fate of adsorbed molecules interacting with paramagnetic surface sites being investigated by EPR, but provide important means to compare to known properties of the systems under consideration. IR spectroscopy in reflection adsorption geometry and scanning tunneling microscopy (STM) are available to study these properties. The combination of these techniques together with the requirements posed by the EPR spectroscopy dictates the design of the setup. In particular, it is not possible to have both the preparation capabilities as well as the mentioned analytics within one ultrahigh vacuum chamber. As shown in Figure 1 the system is split into two main chambers, the preparation chamber shown on the right, which also contains the IR spectrometer (blue), and the analysis chamber on the left, which hosts the EPR spectroscopy (red) and STM (green). In between the two main chambers a small high-pressure cell with a load lock system is attached and separated from the other chambers by gate vales (VAT). The entire setup is placed on a $2 \times 3 \mathrm{~m}^{2}$ large nonmagnetic optical bench (Newport RS3000), which rests on active vibration isolation units (HWI Scientific Instruments, AVI 400).

Within this section we will describe the design of the different parts of the system starting with a description of 


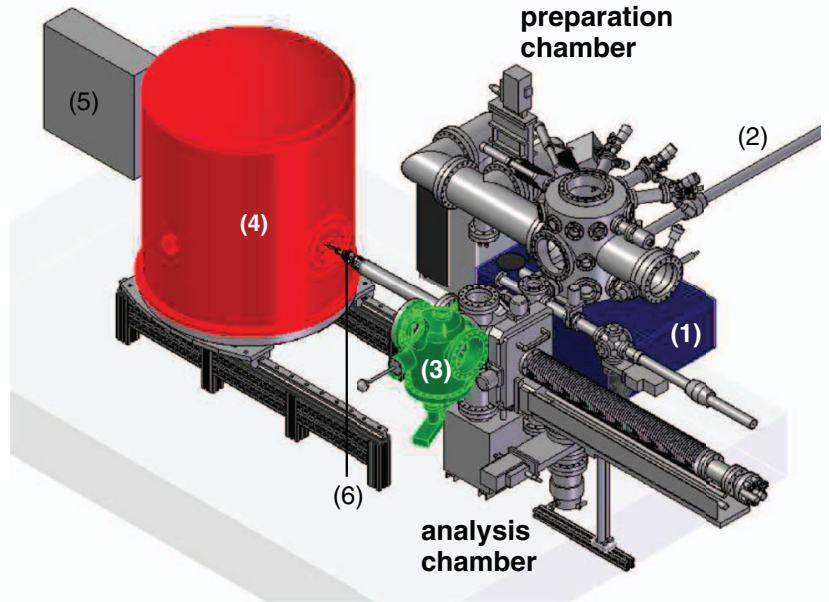

FIG. 1. Schematic view of the experimental setup. The preparation chamber is shown on the right (vertical manipulator is omitted for clarity) and the analysis chamber on the left. (1) IR spectrometer (blue), (2) transfer rod, (3) STM (green), (4) superconducting magnet (red), (5) microwave bridge, and (6) resonator.

the preparation chamber in Subsection II A followed by a discussion of the design of the analysis chamber in Subsection II B. The implementation of the EPR spectrometer in particular the design of the resonator and the various aspects that are required for a stable operation of the experiment will be described in Sec. III.

\section{A. Preparation chamber}

The preparation chamber consists of three levels of flanges and a small IR cell mounted at the bottom of the chamber shown in Figure 2. A vertically mounted, motorized manipulator (VAb) with $600 \mathrm{~mm}$ travel length (1) allows to trans-

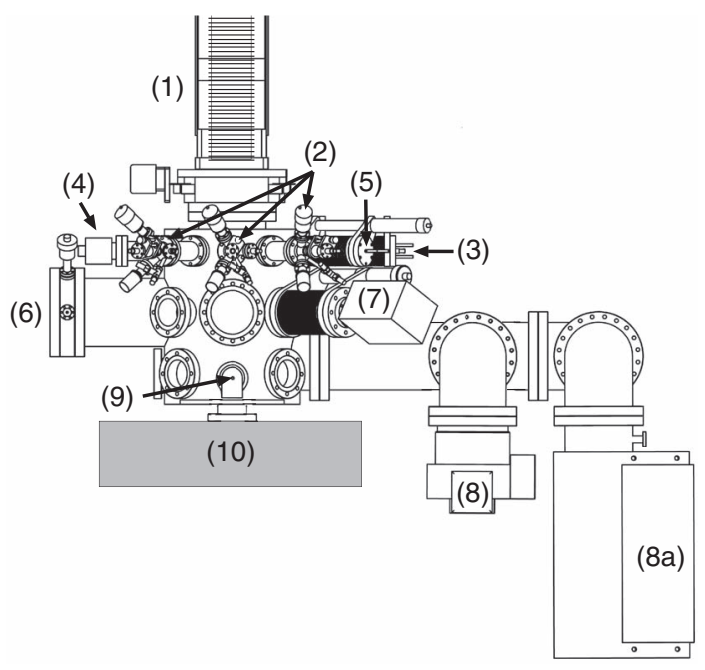

FIG. 2. Side view of the preparation chamber looking along the transfer rod towards the analysis chamber (see Fig. 1). (1) Vertical manipulator, (2) electron beam evaporators, (3) quartz microbalance, (4) sputter gun, (5) pin hole doser, (6) LEED/Auger optics, (7) quadrupole mass spectrometer, (8) turbo molecular pump, (8a) ion getter pump, (9) magnetic transfer rod, and (10) IR spectrometer. fer the sample between the levels. The manipulator houses a helium flow cryostat (KONTI Cryostat, CryoVac).

The topmost level of the chamber carries up to three electron beam evaporators (2) (Omicron, EFM3 or triple EFM) for deposition of metals, which can be calibrated by a quartz microbalance (3) (QMB, Intellemetrics, IL 150). An ion gun (4) (DPIG, NGI 3000-Se, LK Technologies) is affixed to clean the single crystal surfaces. A pin-hole gas doser (5) completes this level. The construction of the pin hole doser is described in detail in Ref. 27. The second level comprises a combined low energy electron diffraction (LEED)/Auger system (6) (Spectaleed, Omicron) to check for long-range order and chemical composition of the surface. In addition, a quadrupole mass spectrometer (7) (HIDEN, Hal 201) is attached, which allows to control the residual gas composition as well as temperature programmed desorption experiments. The third level carries the vacuum gauge, the connection to the pumping stack consisting of turbo molecular (8) (Oerlicon, Turbovac 361), ion getter (8a) (IGP, Varian, 3001 star cell), and titanium sublimation pump (TSP, not shown), as well as the magnetic transfer rod (9) (MLRM, VG scientific) to move the sample between the different chambers. The transfer functionality is based on the Omicron transfer mechanism as sample plates based on the Omicron design are used to mount the sample.

A small UHV chamber is attached to the bottom of the preparation chamber to allow for infrared measurements in reflection absorption geometry. The design has been described in detail in Ref. 27. In short, the UHV chamber comprising two $\mathrm{KBr}$ windows (Korth, Kiel) at an angle of $170^{\circ}$ is lowered into the internal sample compartment of the vacuum FTIR system (10) (IFS 66v/S, Bruker), which allows to use the internal MCT detector of the spectrometer. A plate connected to the UHV chamber by a flexible bellow is used to seal the sample compartment of the FTIR spectrometer. A pin hole gas doser using the same principle discussed above, however, adapted to the space requirements imposed by the IR spectrometer is mounted to the UHV chamber to allow for gas adsorption in the measurement position. Because of the tight space requirements and the need to minimize the pumped volume of the pin hole doser pneumatically driven valves (Swagelok, BK series) operated by a home made electronics are used to control gas inlet and pumping of the doser.

One has to bear in mind that the preparation chamber is situated with less than $1.5 \mathrm{~m}$ distance to the superconducting magnet typically operated at about 3.5 T during EPR experiments. As the stray field of the magnet at the site of the preparation chamber is significant and very inhomogeneous this has consequences for both instrumental techniques using charged particles such as LEED/Auger or ion gauge as well as technical equipment such as the turbo molecular pump. These restrictions imply that the magnetic field used for EPR is only switched on shortly before EPR measurements. In case the magnetic field is present the TMP has to be switched off and the pressure in the preparation chamber is maintained by the IGP and the TSP only. The positioning of the pumps was chosen such as to maximize the distance to the superconducting magnet. This is particularly important for the IGP as the permanent magnets of the pump interact with the stray field of 


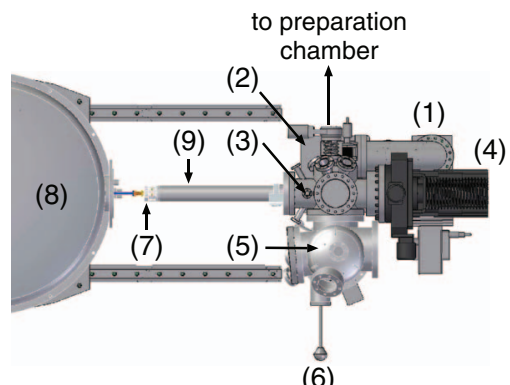

(6)

FIG. 3. Top view of the analysis chamber. (1) Turbo molecular pump, (2) ion getter pump, (3) filament for electron bombardment of the sample, (4) long travel manipulator, (5) scanning tunneling microscope, (6) pincer grip wobble stick, (7) resonator housing made out of Macor, (8) superconducting magnet, and (9) titanium tube.

the magnet and can cause the He-reservoir inside the magnet to move and create a thermal leak, which results in a sharp increase of helium consumption, if placed too close to the magnet.

\section{B. Analysis chamber}

The main purpose of the analysis chamber is to allow EPR as well as STM experiments of the prepared samples. The chamber (Pink) shown in Figure 3 is pumped by a stack containing a turbo molecular (1) (Pfeiffer TMP260), an ion getter (2) (Varian, star cell 300 1), and a titanium sublimation pump attached to the bottom of the chamber. The TMP can be separated from the chamber by a pneumatically driven gate valve (MDC) and is surrounded by a magnetically soft iron housing to shield the pump from the stray field of the magnet and allows operating the pump with the magnetic field being present. The chamber further contains a leak valve for background dosing and an ion gauge for pressure reading (not shown). A filament for annealing and electron bombardment (3) is mounted on a rotation feed through (not shown) to place it in front of the sample. Viewports assure safe sample handling. The sample is moved in and out of the analysis chamber by the transfer rod attached to the preparation chamber (see Sec. II A). The sample is transferred onto the horizontally mounted manipulator (4) (Pink) with $700 \mathrm{~mm}$ traveling length, which houses a specially configured He flow cryostat (KONTI Cryostat, CryoVac). The sample is placed in a sample holder mounted at the end of the He-cryostat such that the surface to be investigated is oriented perpendicular to the long axis of the manipulator. A variable temperature scanning tunneling microscope (5) (VT STM, Omicron), which has a storage carousel for up to nine samples and tips, is attached to the side of the chamber. The current implementation only allows room temperature measurements due to the requirements of the sample setup. The sample that is mounted on standard Omicron sample plates made out of molybdenum is transferred between the STM, storage carousel, and manipulator by a pincer-grip wobble stick from Omicron (6).

The traveling length of the manipulator was chosen such that sample transfer from the preparation chamber and the positioning of the sample in the center of the superconducting magnet (8) during measurements is possible. The housing of the Fabry-Perot resonator (7) is made out of Macor and attached to a Ti-tube (9). The latter is mounted to the chamber opposite of the manipulator and can be inserted into the horizontal bore (ID $60 \mathrm{~mm}$ ) of the superconducting magnet, whose magnetic field is oriented horizontally and orthogonal to the long axis of the titanium tube. Titanium was chosen here to ensure a low relative magnetic permeability $\left(\mu_{\mathrm{r}}\right.$ $<1.001)$ for the tube being located in the center of the magnet. The Fabry-Perot resonator resides at the very end of the tube. The details of the resonator design will be discussed later (Sec. III). At this point it is important to realize that pressures in the low $10^{-10}$ mbar range - necessary to study surfaces under well-defined conditions - require the chamber to be baked to $100-150{ }^{\circ} \mathrm{C}$. Heating the chamber cannot be done inside the cryo-magnet. Therefore, the magnet is located on a rail system, which allows a high precision linear motion such that the entire UHV chamber is outside the magnet during bake out and the resonator is positioned in the center of the magnet during the measurements. While this sounds like a simple task, technically it requires some effort, because of the tight space requirements imposed by the $60 \mathrm{~mm}$ wide bore of the magnet. To maximize the space inside the Ti-tube it has a $54 \mathrm{~mm}$ outer and $50 \mathrm{~mm}$ inner diameter. This implies that the alignment of magnet and chamber needs to be better than $0.1^{\circ}$ if the symmetry axis of both the Ti tube and bore of the magnet are aligned better than $1 \mathrm{~mm}$ in both dimensions. As the guiding tube of the manipulator, which houses the Hecryostat, has an outer diameter of $44 \mathrm{~mm}$, it also requires that the long axis of the manipulator and the Ti-tube axis have to have a relative inclination smaller than $0.1^{\circ}$. In addition, to the precision of the $\mathrm{Ti}$ tube and the manipulator, it also poses particular requirements with respect to the parallelism of the flanges (better than $0.1^{\circ}$ with respect to each other) to which the manipulator and the titanium tube are connected.

The performance of the EPR spectroscopy is strongly governed by the mechanical properties of the setup. Apart of the alignment of the sample the stability against vibrations plays a crucial role. The sample is mounted at the end of the He cryostat, which is moved by $700 \mathrm{~mm}$ into the center of the superconducting magnet. This creates a long lever, which is prone to pick up vibrations present in the system. While the system is isolated against vibrations of the floor by the active vibration isolation system, acoustic noise is an issue. To reduce the impact of vibrations a sample setup as shown in Figure 4 was installed.

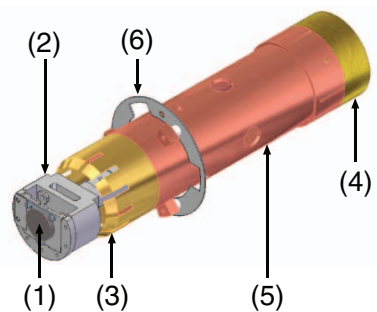

FIG. 4. Configuration of the sample setup on the manipulator in the analysis chamber. (1) Sample on sample plate, (2) sample holder, (3) bronze spring, (4) cooling shield of the He-cryostat connected with the secondary heat exchanger, (5) $\mathrm{Cu}$-tube used as cooling shield and stabilization element, and (6) electrode to ensure proper alignment of the sample inside the Ti tube. 
As mentioned above the sample (1) is mounted onto the face side of the long travel manipulator by means of a Mo sample holder (2) connected to the primary heat exchanger of the He cryostat. The sample is pressed onto the Mo sample holder by Mo-springs. The vibration isolation of the long lever is achieved by means of a circular bronze spring (3) attached via a Cu-tube (5) to the secondary heat exchanger of the He-cryostat (4) and fixed against the sample holder by set screws. The bronze spring fits into the Macor body used as a housing for the Fabry Perot resonator discussed in detail below. Because of the very poor thermal conductivity of Macor the thermal contact of the cooled bronze spring has no measurable effect on the sample temperature. In addition, a metal electrode (5) electrically isolated from the rest of the setup is mounted to the $\mathrm{Cu}$-tube. This electrode is used to probe the positioning of the cryostat inside the Ti-tube. To this end the resistance between the electrode and the chamber is controlled while the sample is transferred into the measurement position. This is particularly crucial as there is no direct visual access into the Ti tube from the outside.

\section{IMPLEMENTATION OF THE EPR SPECTROMETER}

\section{A. The design of the Fabry-Perot resonator}

As mentioned in the Introduction the key design idea of the current experiments is the use of the metal single crystal being the substrate for the heterogeneous model catalyst as one mirror of a Fabry-Perot resonator while the other side is a curved mirror connected through a coupling orifice, and subsequent wave guide elements to the microwave bridge. It is the design of this resonator, which determines the performance of the experiment crucially. A key problem that has to be addressed is the compatibility of cw-EPR spectroscopy and ultrahigh vacuum conditions. cw-EPR spectroscopy is typically detected using lock-in technology. Therefore, the static magnetic field is modulated, e.g., by a set of Helmholtz-coils. The requirement to couple an $\mathrm{AC}$ magnetic field into the UHV impedes the use of a metal chamber. Figure 5 shows the implementation of the FP resonator schematically. The FPresonator assembled in situ by the sample (1) and the $\mathrm{Cu}$ mirror (2) is placed inside of a Macor block (3), which is mounted to the end of the Ti-tube (4) being attached to the analysis chamber (see Sec. II B). The sample with a diameter
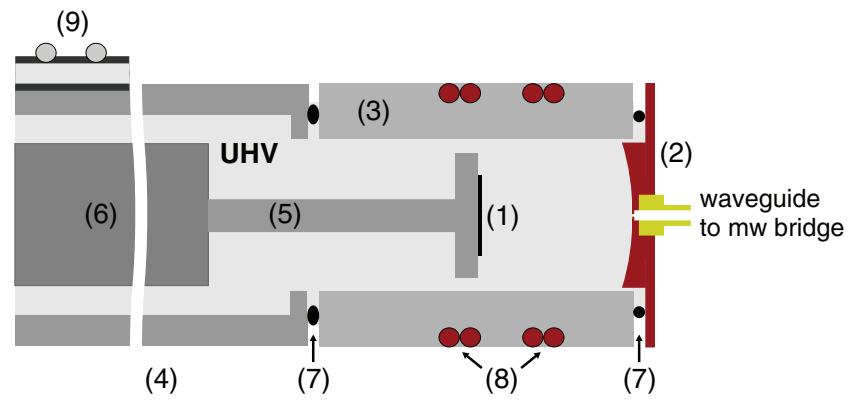

FIG. 5. Schematic drawing of the Fabry Perot resonator. (1) Sample; (2) Cumirror; (3) Macor body; (4) Ti-tube connected to the UHV chamber; (5) sample holder; (6) He-cryostat; (7) Viton sealing; (8) Helmholtz coils for modulation of the static magnetic field; and (9) Peltier element. of $12 \mathrm{~mm}$ is mounted on an Omicron sample plate to ensure compatibility to the STM. The size of the sample is limited by the sample plate and the space required to ensure a proper transfer of the sample plate. The sample plate is inserted into the sample holder (5), which is connected to the He-cryostat (6). This design allows for a cooling of the sample to approximately $50-70 \mathrm{~K}$. The lowest possible temperature is determined by the surrounding radiation field and depends on the details of the sample set up. The sample plate is electrically isolated against ground by means of a sapphire plate placed within the sample holder, which ensures good thermal contact at low temperature. The isolation allows to put the sample onto a voltage if the sample is, e.g., to be bombarded with electrons. The outer part of the Cu-body is used as a flange and sealed against atmosphere by means of a Viton ring (7) residing in a corresponding notch of the Cu-body. A Viton seal is also used for the connection to the Ti-tube. The inner part of the Cu-body with a diameter of $28 \mathrm{~mm}$ is used to create the curved mirror of the FP-resonator. A pair of Helmholtz coils (8) (Müller Spulenwickeltechnik, Reichshof) glued onto the outer walls of the Macor body is used for modulating the static magnetic. Due to the large distance in comparison with the possible size of the coils the modulation field on the sample is restricted to a few Gauss, without significant perturbation due to microphonic noise.

The target pressure for the experiments in the analysis chamber is $1 \times 10^{-10} \mathrm{mbar}$. While this can be achieved by the Viton seals described above the most critical part is the sealing of the microwave source in an UHV compatible manner. During the development of this machine different approaches have been explored. The first approach was based on using a quartz window inside the resonator to decouple the UHV from atmosphere. This approach is inspired by the implementations at X-Band where a quartz tube inserted into a standard cavity operating at atmospheric pressure is used. Such a design benefits from the excellent vacuum properties of quartz. At W-band the situation is more complex as quartz absorbs microwave radiation at $94 \mathrm{GHz}$. Hence the window has to be chosen as thin as possible to reduce dielectric losses $(150 \mu \mathrm{m}$; diameter $30 \mathrm{~mm}$ ). A thin quartz window, however, is mechanically not stable enough to withstand the force imposed by atmospheric pressure. Hence, the backside of the window and the rest of the Fabry Perot resonator have to be situated in at least fine vacuum ( $p<1$ mbar). These requirements render the operation of the setup complicated. In particular, pumping down and venting of the apparatus requires particular care as pressure differences beyond a few mbar lead to destruction of the window. More importantly, the presence of a window inside the Fabry Perot resonator causes severe problems with the reproducibility of the measurements, which were traced back to small changes in the geometric structure of the Fabry Perot resonator beyond the mechanical precision of the components available. For subsequent measurements changes in the background of the resonator occur, if the sample was removed from the measurement position, which is necessary to perform subsequent preparation steps, and placed at nominally the same position. This impedes the measurement of small EPR signals from thin film samples, because of the small number of paramagnetic sites expected for such 


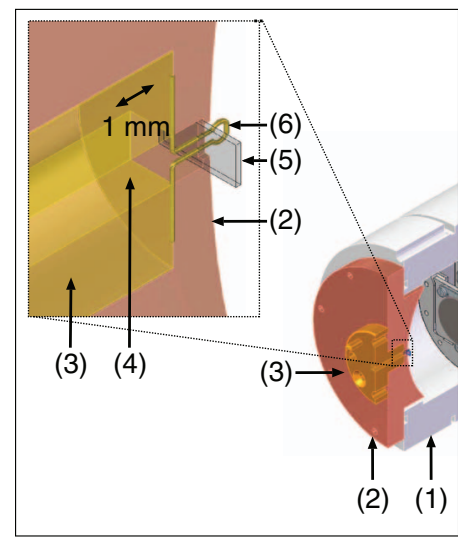

FIG. 6. Detailed view on the coupling orifice and the vacuum sealing. (1) Macor body; (2) Cu-mirror; (3) brass fitting; (4) WR 28 waveguide; (5) sapphire plate; and (6) coupling antenna made out of $60 \mu \mathrm{m} \mathrm{Au}$ wire.

samples. Background stability is a key factor for these investigations as EPR signals from the sample have to be discriminated from background signals of the setup by proper correction of the data.

Despite the difficulties in terms of background stability the coupling structure of the resonator using an antenna with an optimized coupling orifice, which was developed for the quartz sealed resonator, turned out to be stable and robust. Therefore, the coupling structure, which has been adapted from a development at $48 \mathrm{GHz}$ reported in literature, should be preserved. ${ }^{28}$ The right side of Figure 6 shows the very end of the current design. It consists of the Macor body (1) and the Cu-Mirror (2) together with a brass fitting (3), which is inserted into the $\mathrm{Cu}$-mirror. The brass fitting is made of a short piece of WR28 $\left(1.27 \times 2.54 \mathrm{~mm}^{2}\right)$ waveguide (4) ending in a modified UG387/U flange. Thus the fitting allows to connect the $\mathrm{Cu}$-mirror with the microwave bridge which is realized by an oversized waveguide of approximately $1 \mathrm{~m}$ length to reduce losses of the transfer line.

The coupling of the microwave is achieved by means of an antenna made out of $60 \mu \mathrm{m}$ thick Au wire. The antenna is mandatory to couple microwave radiation through the small rectangular coupling orifice with an opening of $200 \times 900 \mu \mathrm{m}^{2}$. The wire is pressed between the end of the Brass fitting and the $\mathrm{Cu}$-mirror. The distance between the wire and the coupling orifice is $\lambda / 4$ to maximize the pickup of the displacement currents, which are used to transmit the radiation into the resonator. The structure in the $\mathrm{Cu}$ mirror consists of a rectangular hole to fit the WR 28 waveguide part of the brass fitting, the piece with a length of $\lambda / 4$ and the inner dimensions of a WR 28 waveguide as well as the coupling orifice. All these have to be manufactured with very high precision to ensure a proper press fit of the coupling wire, a perfect alignment of the waveguide pieces (brass and copper) and an orifice with as small a wall thickness as possible to reduce losses.

Based on the experience with the quartz window it is clear that the vacuum sealing of the microwave source has to be shifted outside of the resonator. To this end the options are very limited as the coupling orifice in the curved mirror is too small to allow for efficient pumping of any volume that might be enclosed behind it. Therefore, the seal has to be placed directly on the backside of the coupling orifice. A $1.1 \times 2.3 \mathrm{~mm}^{2}$ large and $150 \mu \mathrm{m}$ thick sapphire plate (Dopa $\mathrm{GmbH}$, Berlin) fitting into a WR28 waveguide is glued onto the backside of the $\mathrm{Cu}$ mirror using a UHV compatible glue (Varian TorrSeal, Agilent Technologies). The Au antenna is fed into the resonator by two $300 \mu \mathrm{m}$ wide holes separated by $300 \mu \mathrm{m}$, which have to be centered inside the coupling orifice as shown on the left hand side of Figure 6. TorrSeal is used to glue the antenna into the holes of the sapphire window. The glue has to be used with care as TorrSeal absorbs microwaves strongly. Glue should be used thinly and be restricted to the backside of the orifice and the holes, respectively. Extra pieces of glue lead to a strong reduction of the available microwave power inside the resonator. This setup of the coupling structure is mechanically robust and results in a very good reproducibility of the spectra as will be discussed in Sec. IV in more detail. It turned out that the coupling, however, degraded over periods of months in particular if the system was heated several times. This observation was interpreted as a loss of conductivity between the walls of the waveguide (brass/copper interface) and the antenna due to oxidation. It is believed that this process is particularly rapid during bake-out of the system, where the components are heated to $100-150^{\circ} \mathrm{C}$. Therefore, a pumping port was designed, which encloses the atmospheric side of the resonator (copper mirror (2) and brass fitting (3) in Fig. 6) after removing the other waveguide parts and is evacuated to $10^{-2}$ mbar during bake-out. This procedure results in a stable setup.

Optimal coupling of the microwaves into the resonator requires an impedance matching between the resonator and the microwave line. For a collinear arrangement of waveguide and resonator a so-called three-screw tuner can realize that. Figure 7 shows the design of the current implementation, which can be mounted directly onto the $\mathrm{Cu}$-mirror by the UG387/U flange (1). Three screws (2) lined up along the longitudinal direction of the waveguide (3) are used. The distance between the screws used in literature varies between $3 \lambda_{w g} / 8$ and $5 \lambda_{w g} / 8 .{ }^{29}$ Due to the small dimensions of the WR 28 waveguide a distance of $5 \lambda_{\mathrm{wg}} / 8$ was chosen. Metal rods of 0.25 and $0.5 \mathrm{~mm}$ diameter and a length of up to $1.25 \mathrm{~mm}$ turned down from M1 brass screws were used to adjust the coupling. The optimal dimensions for the stubs are determined experimentally. In a first step M1 threads were tapped into the wall of the W-band waveguide. The tolerance of the thread gives rise to mechanical instabilities, which were reduced by Viton tubes between the screw heads and the

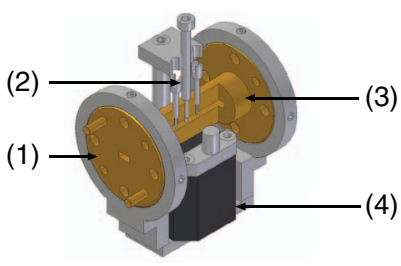

FIG. 7. Drawing of a three-screw coupler. (1) UG387/U flange, (2) adjustable brass rods, (3) WR 28 waveguide, and (4) piezo motor (Attocube ANPz51). 
waveguide acting as spring loads. It was found that the optimum set of screws (diameter, length of the stubs) depends on the details of the antenna used. Therefore, the tuner setup is adjusted for every new antenna. In most cases the influence of one screw onto the coupling was highest. A complication arises from the fact that the details of the coupling depend on the position of the magnet, which is due to small mechanical changes induced by moving the heavy magnet on the optical bench. However, the screws are not accessible if the magnet is in the measurement position. Therefore, one screw was replaced by a rod mounted to a linear piezo motor (4), which allows a precision movement with the magnet in measurement position. For the current design with a $60 \mu \mathrm{m} \mathrm{Au}$ antenna and a $150 \mu \mathrm{m}$ thick sapphire window the resonator is only slightly undercritically coupled. Hence, the effect achieved by the coupler on the resonator performance is small and the coupler can also be omitted.

\section{B. Characterization of the resonator}

For a Fabry Perot resonator operated in a $\mathrm{TEM}_{00 \mathrm{n}}$ mode the radius of curvature of the mirrors controls the size of the Gaussian beam waist. In the design with one planar and one curved mirror the beam waist is located on the surface of the planar mirror. Therefore, it is desirable to maximize the size of the Gaussian beam waist if paramagnetic species on the planar mirror should be investigated. Increasing the size of the beam waist, however, reduces the $\mathrm{Q}$ factor of the resonator and will reduce the sensitivity of the setup. In a first set of experiments mirrors with different radius of curvature were tested. In literature a variety of Fabry Perot resonators have been reported. In these setups radii of curvature in the range of $15-17 \mathrm{~mm}$ were used. These radii were chosen to avoid the high mode degeneracy that is expected for a confocal or in this case semi-confocal arrangement of the mirrors. Characterization of such resonators has shown that the beam waist (the radius at which the $\mathrm{B}_{1}$ field has dropped to $1 / \mathrm{e}$ of its initial value) in the center of the resonator is about $3 \mathrm{~mm}$, which can serve to estimate the probed sample surface. ${ }^{24}$ As a starting point we have chosen a radius of curvature of $17 \mathrm{~mm}$ as it allows to place the resonator in a near semi-confocal arrangement, which maximizes the beam waist on the planar mirror. The performance of resonators employing mirrors with different radius of curvature was tested using EPR spectra taken at room temperature for a DPPH (2,2'-diphenyl1-picrylhydrazil, Sigma Aldrich) sample prepared on an aluminum disc (diameter $12 \mathrm{~mm}$ ). The measurements were done under atmospheric conditions. An important prerequisite of the sample is the homogeneous distribution of the spins on the surface of the disc to ensure that a change of the microwave distribution and corresponding possible changes of the signal can be related to the area sensitivity of the setup. The sample was prepared by spin coating from a DPPH solution at a rotating speed of $2000 \mathrm{rpm}$. The total amount of spins dropped onto the surface was $1 \times 10^{16}$ spins $/ \mathrm{cm}^{2}$, however, the density of molecules has not been determined quantitatively and significant losses are expected due to the spin coating process. Figure 8 shows the signal to noise ratios measured for four mirrors with radius $17,20,25$, and $30 \mathrm{~mm}$ using the

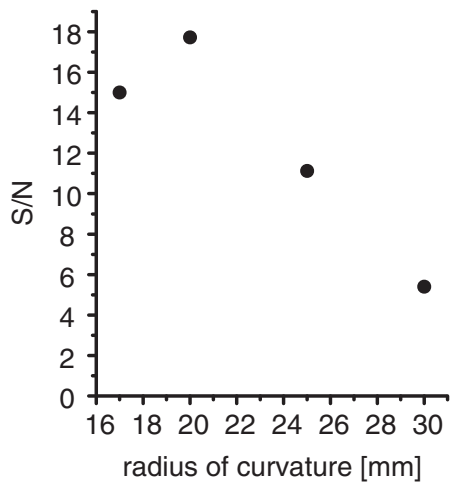

FIG. 8. Signal to noise ratio as determined for a DPPH sample on a $12 \mathrm{~mm}$ diameter $\mathrm{Al}$ disc. Spectra are taken under atmospheric conditions at room temperature.

same sample prepared as described above. Each mirror was equipped with its own antenna and tests with different antennas for each mirror were done independently to reduce the error that may arise from the fact that the antenna arrangement deviates for each setup. As seen from Figure 8 the signal to noise ratio is found to be lowest for the mirrors with a radius of $30 \mathrm{~mm}$ and increases by about a factor of 3 if the radius is reduced to $20 \mathrm{~mm}$. The mirror with a radius of $17 \mathrm{~mm}$ shows a slightly reduced signal to noise ratio. However, the $10 \%$ difference is not significant given the experimental uncertainties discussed above. The observed $\mathrm{S} / \mathrm{N}$ ratios scale well with the quality factor of the resonator indicating that losses due to a larger beam waist cannot be compensated for by an increased beam waist, hence an increased number of paramagnetic centers being irradiated on the surface. From these results it is concluded that radii of curvature around $20 \mathrm{~mm}$ are optimal for the given sample size of $12 \mathrm{~mm}$.

For a given mirror the performance of the resonator depends also on the resonance mode. Typical Q values between 2500 and 3000 are achieved with this setup. The highest sensitivity is found for a distance of about $9.5 \mathrm{~mm}$ between the mirrors corresponding to a $\mathrm{TEM}_{006}$ mode. Adjacent modes with both larger and smaller mirror distances were found to show reduced signal to noise ratios for the test samples described above.

As discussed in Sec. II B the sample is mounted at the end of a He flow cryostat while the curved $\mathrm{Cu}$-mirror is rigidly connected to the UHV chamber. This results in a different thermal coupling of the two mirrors with the laboratory environment. In turn temperature changes in the laboratory cause a displacement of the $\mathrm{Cu}$-mirror relative to the sample due to thermal expansion of the Ti-tube. Using the thermal expansion coefficient of $\mathrm{Ti}(8.5 \mu \mathrm{m} /(\mathrm{m} \mathrm{K}) \text { at room temperature })^{30}$ and a length of the Ti tube of $50 \mathrm{~cm}$ a displacement of $425 \mathrm{~nm}$ is expected for a temperature change of $0.1 \mathrm{~K}$. This corresponds to a shift in frequency of about $4 \mathrm{MHz}$. While such a displacement can be compensated by the automatic frequency control (AFC) of the microwave bridge (dynamic range of $6 \mathrm{MHz}$ ) slightly larger displacements will exceed the dynamic range of the AFC, which causes significant problems with the stability of the spectrometer. For the sample being at room temperature the dynamic range of the AFC is sufficient to stabilize the system on the time scale of minutes. This time 


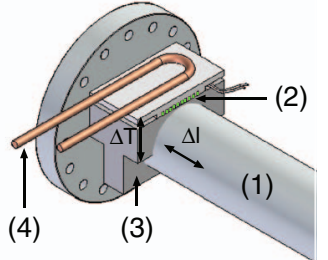

FIG. 9. Schematics of the Peltier element used to control the length of the Ti tube. (1) Ti-tube, (2) Peltier element, (3) Al collar, and (4) water cooling.

decreases significantly if the sample is cooled by liquid helium. The time scale of stable operation is considerably shorter than the one required for the measurements of paramagnetic species of the thin oxide film samples. For samples with less than $10^{13}$ spins $/ \mathrm{cm}^{2}$ the accumulation time of the spectra required to have sufficient signal to noise ratio is typically several hours. Hence, a stabilization mechanism for the resonator is required.

Controlling the temperature of the laboratory to the degree required for a stable operation of the resonator is beyond reach. Hence, the distance between sample and $\mathrm{Cu}$-mirror needs to be actively controlled. This involves two aspects. On the one hand the distance has to be changed and on the other hand it needs to be measured and both have to be done with accuracy in the range of less than $100 \mathrm{~nm}$. A variety of approaches have been tested to change the distance between the mirrors including an active control of the sample position on the cryostat head using piezo driven motors. The most reliable solution is shown in Figure 9. The strategy is to actively control the length of the Ti tube (1) by changing its temperature. To this end a Peltier element (2) is connected to the Ti tube by means of an Al-collar (3). Water (4) is used to thermalize the Peltier element from the other side. Due to the heat capacities of the components involved the time constant of this device is tens of seconds. It was already indicated that the thermal drift observed in the measurements without active control involves time scales in that range rendering this approach applicable.

Still the dynamic range of this device is limited and the system has to be close to the thermal steady state for this compensation device to work properly. This is particularly important for measurements at low temperature, because thermal equilibration of the sample position requires considerably more time than cooling down the sample. While the operating temperature at the cryostat head is typically reached within $30 \mathrm{~min}$, it takes about $2 \mathrm{~h}$ for the cryostat to reach its steady state.

As mentioned above the second aspect is to measure and control the distance of the mirrors. To this end it turned out that using the AFC signal of the microwave bridge is the simplest way to achieve this. The AFC signal is provided via an auxiliary output on the backside of the X-band bridge and is fed into a PID controller realized in software using Labview. A careful choice of the PID parameters allows stabilizing the AFC level for tens of hours, provided that the temperature at the primary heat exchanger of the He cryostat is stable, too. Routinely data acquisition for time spans only limited by the amount of liquid helium available to cool the sample can be realized using this setup.

\section{MEASUREMENTS UNDER UHV CONDITIONS}

Initial tests of the setup under UHV conditions were done using di-tert-butyl nitroxide (DTBN) a stable volatile nitroxide radical. A $20 \mathrm{ML}$ thick $\mathrm{MgO}(001)$ film grown on a Mo(001) single crystal surface was chosen as the substrate to avoid suppression of the EPR-signal of molecules in direct contact to the metal surface, which leads to a loss of the EPR signal due to spin exchange with the conduction electrons. ${ }^{31,32}$ Quenching of an unknown amount of adsorbed molecules would hamper the estimation of the sensitivity of the Fabry-Perot resonator. It was shown for an alumina film grown on $\mathrm{NiAl}(110)$ that a $5 \AA$ thick film is already sufficient to decouple the unpaired electron from the conduction band electrons at the Fermi level and observe submonolayer quantities of DTBN. ${ }^{33}$ The experiments were performed at a base pressure $<2 \times 10^{-10}$ mbar cooling the sample to $70 \mathrm{~K}$ as measured with a type $\mathrm{C}$ thermocouple. Liquid DTBN purchased from Aldrich (300721, melting point $-6^{\circ} \mathrm{C}$, purity 90\%) stored in a glass vial was attached to the chamber via leak valve. Prior to use DTBN was purified using several freeze-thaw cycles.

DTBN reacts readily with the walls of the chamber. Therefore, the UHV chamber was passivated by backfilling with DTBN at a pressure of $1 \times 10^{-7}$ mbar for $600 \mathrm{~s}$ prior to adsorption on the $\mathrm{MgO}$ surface. Afterwards the EPR cryostat was cooled down to helium temperature. Prior to the experiment the $\mathrm{MgO}$-film was annealed to $700 \mathrm{~K}$ in the preparation chamber to remove remaining adsorbates and then transferred into the analysis chamber. The top spectrum in Fig. 10 shows the EPR spectrum taken after adsorption of $3 \mathrm{~L}$ (1 L (angmuir) corresponds to $10^{-6}$ torr $* \mathrm{~s}$ ) of DTBN at $70 \mathrm{~K}$. The spectrum is dominated by a single line centered at $\mathrm{g}=2.006$ with a peak to peak line width of $1.8 \mathrm{mT}$. After this spectrum was taken the sample was transferred back to the preparation chamber and heated to $700 \mathrm{~K}$, beyond which no desorption of molecular fragments was observed by mass spectrometry in line with results on an alumina film grown on $\mathrm{NiAl}(110){ }^{33}$ After transfer into the analysis chamber $0.38 \mathrm{~L}$ DTBN was dosed and spectrum (b) was obtained. The spectrum shows a line at slightly higher field $(\mathrm{g}=2.0054)$, which exhibits a significantly increased line width of $3.4 \mathrm{mT}$. Finally, the

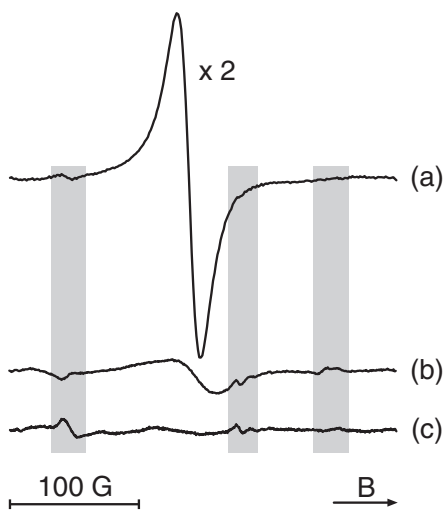

FIG. 10. W-band EPR spectra taken for DTBN adsorbed on $20 \mathrm{ML}$ $\mathrm{MgO}(001) / \mathrm{Mo}(001)$. (a) $31 \mathrm{DTBN} @ 70 \mathrm{~K}, 50$ scans, (b) sample from (a) heated to $700 \mathrm{~K}$ and adsorption of 0.381 DTBN @ 70 K, 200 scans, and (c) sample from (b) after heating to $700 \mathrm{~K}, 200$ scans (for details see text). 
annealing step in the preparation chamber was repeated and a background spectrum (c) was recorded, which lacks the signal related to DTBN.

The g-value of the line observed after absorption of $3 \mathrm{~L}$ DTBN agrees well with values found in literature (2.0056 on alumina, ${ }^{34} 2.0058$ on oxidized copper ${ }^{35}$ ). The line width is governed by exchange narrowing, which depends on the arrangement of the molecules on the surface as demonstrated by the strong temperature dependence of the line width observed for multilayer adsorption of DTBN on a thin alumina film under UHV conditions. ${ }^{33}$ At X-band line widths ranging from 1 to $3.5 \mathrm{mT}$ have been observed depending on the preparation conditions, which agrees well with the observed line width of $1.8 \mathrm{mT}$.

The broadening of the signal for $0.38 \mathrm{~L}$ coverage cannot be attributed to temperature, instead the reduction of the exchange interaction is due to the reduced absolute number of molecules interacting on the surface and a resulting change of the intermolecular interactions, which can also account for the observed g-shift as compared to the spectrum at higher coverage. The number density of DTBN molecules on the surface can be determined using kinetic gas theory leading to $4.55 \times 10^{14}$ and $5.69 \times 10^{13}$ spins $/ \mathrm{cm}^{2}$ for $3 \mathrm{~L}$ and $0.38 \mathrm{~L}$, respectively. Comparing these number densities to the density of oxygen sites on the surface of $\mathrm{MgO}(001)(1.12 \times$ $10^{15} \mathrm{O}$-atoms $/ \mathrm{cm}^{2}$ ) indicates that the small amount of DTBN corresponds to almost $1 / 20$ of a monolayer. For submonolayer quantities it is reasonable to assume that exchange interaction between molecules is reduced in comparison to the multilayer leading to reduced spin-spin interactions. Double integration of the EPR signal taking the small background contribution into account gives an intensity ratio between the coverages of 5:1. This is smaller than the ratio of $8: 1$ expected based on the nominal exposure of the molecules. The discrepancy may in part be explained by the uncertainty of the precise shape of the baseline, which becomes more important the smaller and wider the signal gets. Additionally, it is possible that DTBN does react with the pristine $\mathrm{MgO}(001)$ surface, which is supported by the observation that inverting the order of adsorption (small dosage first, large dosage second) leads to no or an almost vanishing EPR signal for the small dosage. However, reactions with the chamber walls cannot be excluded despite the passivation procedure performed prior to the initial adsorption. All of these processes decrease the number of spins on the surface such that the given spin densities are an upper limit for the amount being present on the surface. The adsorption of the small amount of DTBN already proves that the setup is sensitive to detect submonolayer quantities of paramagnetic species on well-defined single crystal surfaces. A comparison of the EPR line of DTBN observed here with measurements at X-band mentioned above shows that the sensitivity of setup observed here is comparable with that of the $\mathrm{X}$-band setup. For the latter a sensitivity of $5 \times 10^{11} \mathrm{spins} / \mathrm{cm}^{2}$ was found, which renders the method well suited to detect defect species at low concentrations as required to work with defects to be considered a small perturbation of a surface. ${ }^{36}$

The measurements presented above were obtained from a resonator setup, which uses a quartz window in the resonator to separate UHV from the atmosphere. While the sensitivity of this setup is already very good the small background signals found in the shaded regions in Figure 10 show considerable difference for the different measurements. Qualitatively, the regions behave differently. The lines at low and high field differ rather strongly between the measurements. Even more importantly, the phase of the signals is changing between measurements indicating that the corresponding centers are not associated with paramagnetic species on the surface of the oxide film. The two signals close to the DTBN line are well reproduced in terms of signal position and phase, but the intensity of the features is changing significantly for the various measurements. It is important to note that the observed changes of the background signals were also observed after removing the sample and bringing it back to nominally the same position. For systems with considerably smaller number of spin centers and line width in the same range as the background signal this is detrimental in terms of a reliable interpretation of the results. It is this lack of background stability, which led to the development of the resonator sealing the UHV directly at the coupling orifice as described in Sec. III.

For comparison Fig. 11 shows the EPR spectra obtained for a resonator with a sealing on the backside of the coupling orifice. The spectrum taken at $50 \mathrm{~K}$ from a $30 \mathrm{ML}$ thick $\mathrm{MgO}(001)$-film grown at room temperature on $\mathrm{Ag}(001)$ (red trace) is compared to the spectrum taken from the same film after the sample has been transferred to the preparation chamber, bombarded with electrons of $100 \mathrm{eV}$ and a current of $5 \mathrm{~mA}$. To demonstrate the level of baseline reproducibility three sections of the spectra are enlarged. It is seen that the correspondence of the spectra prior and after electron bombardment is almost perfect over the entire range. Because of the perfect correspondence between the two spectra it is now reliable to use the red spectrum as the background for the black one and interpret the resulting difference spectrum in terms of paramagnetic centers that were produced by the electron bombardment of the thin $\mathrm{MgO}$-film.

From the spectra in Figure 11 it is readily seen that electron bombardment of the $\mathrm{MgO}(001)$ film creates new signals around the free electron $\mathrm{g}$-value. The most prominent line is observed at a g-value of 2.0028. In addition to that signal, additional lines are also found at higher as well as lower

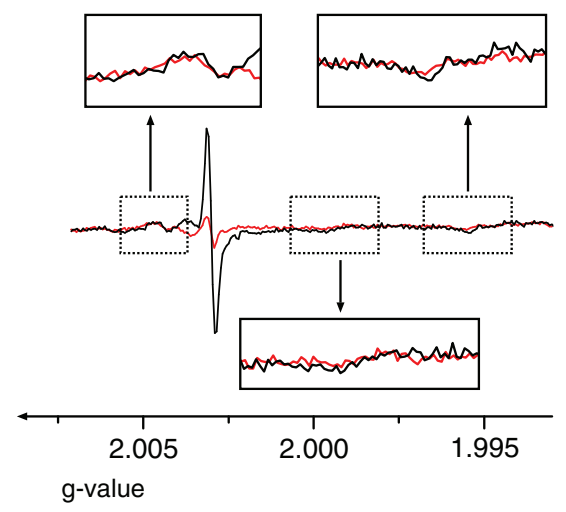

FIG. 11. W-band EPR spectra of a $30 \mathrm{ML}$ thick $\mathrm{MgO}(001)$ film grown on $\mathrm{Ag}(001)$. Red trace: spectrum of the pristine film; black trace: spectrum after electron bombardment. 


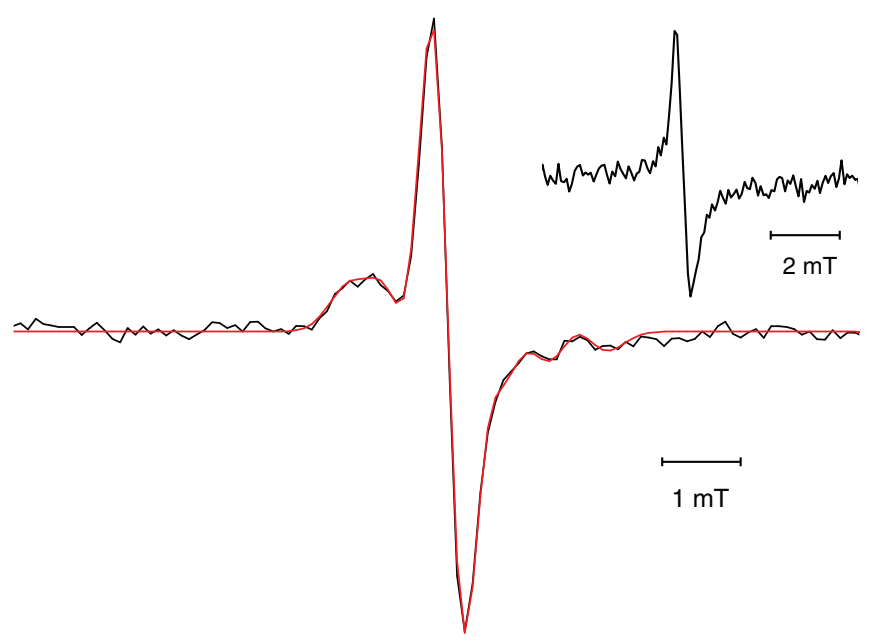

FIG. 12. Background corrected W-band EPR spectrum obtained from subtraction of the spectra in Fig. 11 (black trace); fit to the data assuming 5 Lorentzian lines (red trace); inset: X-band EPR spectrum of a $20 \mathrm{ML}$ $\mathrm{MgO}(001) / \mathrm{Mo}(001)$ prepared at $300 \mathrm{~K}$ annealed to $1100 \mathrm{~K}$ after bombarded with electrons of $100 \mathrm{eV}$.

g-values. While the additional intensity on the low field side is readily seen the high field contribution results in a broadening of the signal. The first question to be addressed is whether the observed signals correspond to surface species. Experiments trying to modify these centers chemically by reaction with molecular oxygen, or $\mathrm{N}_{2} \mathrm{O}$, both proven to react readily with paramagnetic surface centers on $\mathrm{MgO}(001)$ surfaces created by electron bombardment, were unsuccessful. ${ }^{17}$ Hence, it is concluded that the paramagnetic centers observed here are located in the volume of the $\mathrm{MgO}$-films. The latter statement is further substantiated by experiments on the clean Ag surface not shown here. This is in line with observations for $\mathrm{MgO}(001)$ films prepared at RT on $\mathrm{Mo}(001)$ taken at X-band. After electron bombardment a large signal with a line width of about $0.7 \mathrm{mT}$ is observed.

This signal is shown in the inset of Figure 12. The signal is a slightly asymmetric line, however, the resolution at $\mathrm{X}$-band is insufficient to resolve different components that may cause the signal asymmetry. In contrast to that the $\mathrm{W}$ band spectrum observed for the $\mathrm{MgO}(001)$ film on $\mathrm{Ag}(001)$ clearly resolves different spectral components. Together with the measured signal shown in black a fit to the spectrum (red trace) is shown. From the fitting it is found that apart from the central line already discussed above at least four extra components are required to describe the observed line shape. These components have different g-values as compared to the central line ranging from 2.0035 to 2.0018 . While these lines can be resolved at $\mathrm{W}$-band the differences of the $\mathrm{g}$-values are too small to be resolved at X-band hence an unresolved superposition of these lines is observed. It was previously shown that paramagnetic defects in the bulk of $\mathrm{MgO}$ films are trapped electrons located at domain boundaries of the films, ${ }^{37}$ however, a detailed discussion of the different components comprising the spectrum and its comparison to defect centers on $\mathrm{MgO}(001)$ films grown on $\mathrm{Mo}(001)$ go beyond the scope of the article and will be discussed in detail elsewhere. It is, however, important to note that the result presented here clearly proves that the current experimental setup is not only capable to acquire W-band EPR spectra of well-defined oxide films, but also show improved spectral resolution, which allows to distinguish different sites that cannot be differentiated by currently available $\mathrm{X}$-band spectroscopy.

\section{ACKNOWLEDGMENTS}

The authors would like to thank K. P. Dinse, R. Bittl, and A. Schnegg for valuable discussions. The setup of the apparatus was funded through special funds available within the Max Planck Society.

${ }^{1}$ G. Ertl, Angew. Chem., Int. Ed. 47, 3524 (2008).

${ }^{2}$ H.-J. Freund and D. W. Goodman, in Handbook of Heterogeneous Catalysis, edited by G. Ertl, H. Knözinger, F. Schüth, and J. Weitkamp (WileyVCH, Weinheim, 2008)

${ }^{3}$ T. Risse, S. Shaikhutdinov, N. Nilius, M. Sterrer, and H.-J. Freund, Acc. Chem. Res. 41, 949 (2008).

${ }^{4}$ S. Shaikhutdinov and H. J. Freund, Annu. Rev. Phys. Chem. 63, 619 (2012).

${ }^{5}$ G. Binnig and H. Rohrer, Angew. Chem., Int. Ed. Engl. 26, 606 (1987).

${ }^{6}$ B. Henderson and J. E. Wertz, Adv. Phys. 17, 749 (1968).

${ }^{7}$ J. H. Lunsford and J. P. Jayne, J. Chem. Phys. 44, 1492 (1966).

${ }^{8}$ D. Murphy and E. Giamello, J. Phys. Chem. 99, 15172 (1995).

${ }^{9}$ G. Pinarello, C. Pisani, A. D'Ercole, M. Chiesa, M. C. Paganini, E. Giamello, and O. Diwald, Surf. Sci. 494, 95 (2001).

${ }^{10}$ M. Chiesa, E. Giamello, and M. Che, Chem. Rev. 110, 1213 (2010).

${ }^{11} \mathrm{G}$. Hochstrasser and J. F. Antonini, Surf. Sci. 32, 644 (1972).

${ }^{12}$ M. Farle, M. Zomack, and K. Baberschke, Surf. Sci. 160, 205 (1985).

${ }^{13}$ H. Schlienz, M. Beckendorf, U. J. Katter, T. Risse, and H.-J. Freund, Phys. Rev. Lett. 74, 761 (1995).

${ }^{14}$ T. Umeda, M. Nishizawa, T. Yasuda, J. Isoya, S. Yamasaki, and K. Tanaka, Phys. Rev. Lett. 86, 1054 (2001).

${ }^{15}$ M. Yulikov, M. Sterrer, M. Heyde, H. P. Rust, T. Risse, H.-J. Freund, G. Pacchioni, and A. Scagnelli, Phys. Rev. Lett. 96, 146804 (2006).

${ }^{16}$ M. Sterrer, M. Yulikov, E. Fischbach, M. Heyde, H. P. Rust, G. Pacchioni, T. Risse, and H. J. Freund, Angew. Chem., Int. Ed. 45, 2630 (2006).

${ }^{17}$ M. Sterrer, E. Fischbach, T. Risse, and H.-J. Freund, Phys. Rev. Lett. 94, 186101 (2005).

${ }^{18}$ M. Bennati and T. F. Prisner, Rep. Prog. Phys. 68, 411 (2005).

${ }^{19}$ J. H. Freed, Annu. Rev. Phys. Chem. 51, 655 (2000).

${ }^{20}$ O. J. Grinberg, A. A. Dubinskii, V. F. Shuvalov, L. G. Oranskii, V. I. Kurochkin, and J. S. Lebedev, Dokl. Phys. Chem. 230, 923 (1976).

${ }^{21}$ M. A. Ondar, O. Y. Grinberg, A. A. Dubinskii, A. F. Shestakov, and Y. S. Lebedev, Khim. Fiz. 2, 54 (1983).

${ }^{22}$ D. Schmalbein, G. G. Maresch, A. Kamlowski, and P. Höfer, Appl. Magn. Reson. 16, 185 (1999).

${ }^{23}$ H. J. van der Meer, J. A. J. M. Disselhorst, J. Allgeier, J. Schmidt, and W. T. Wenckebach, Meas. Sci. Technol. 1, 396 (1990).

${ }^{24}$ O. Burghaus, M. Rohrer, T. Götzinger, M. Plato, and K. Möbius, Meas. Sci. Technol. 3, 765 (1992).

${ }^{25}$ I. Tkach, U. Rogulis, S. Greulich-Weber, and J. M. Spaeth, Rev. Sci. Instrum. 75, 4781 (2004).

${ }^{26}$ R. T. Weber, J. A. J. M. Disselhorst, L. J. Prevo, J. Schmidt, and W. T. H. Wenckebach, J. Magn. Reson. 81, 129 (1989).

${ }^{27}$ M. Frank, Ph.D. thesis, Humboldt Universität, Berlin, 2000.

${ }^{28}$ U. Harbarth, J. Kowalski, R. Neumann, S. Noehte, K. Scheffzek, and G. Z. Putlitz, J. Phys. E: Sci. Instrum. 20, 409 (1987).

${ }^{29}$ C. P. Poole, Electron Spin Resonance (Dover, Mineola, 1983).

${ }^{30}$ D. R. Lide, Handbook of Chemistry and Physics (CRC Press, Boca Raton, 2005).

${ }^{31}$ U. J. Katter, T. Risse, H. Schlienz, M. Beckendorf, T. Klüner, H. Hamann, and H.-J. Freund, J. Magn. Reson. 126, 242 (1997).

${ }^{32}$ M. Nilges, M. Shiotani, C. T. Yu, G. Barkley, Y. Kera, and J. H. Freed, J. Chem. Phys. 73, 588 (1980).

${ }^{33}$ U. J. Katter, T. Hill, T. Risse, H. Schlienz, M. Beckendorf, T. Klüner, H. Hamann, and H.-J. Freund, J. Phys. Chem. B 101, 552 (1997).

${ }^{34}$ G. P. Lozos and B. M. Hoffman, J. Phys. Chem. 78, 2110 (1974).

${ }^{35}$ M. Nilges and J. H. Freed, Chem. Phys. Lett. 82, 203 (1981).

${ }^{36}$ M. Yulikov, M. Sterrer, T. Risse, and H. J. Freund, Surf. Sci. 603, 1622 (2009)

${ }^{37}$ H. M. Benia, P. Myrach, A. Gonchar, T. Risse, N. Nilius, and H. J. Freund, Phys. Rev. B 81, 241415 (2010) 